Missed Diagnosis

\title{
Angina and myocardial infarction presenting with pain confined to the ear
}

\author{
P.M. Rothwell
}

Department of Medicine, Falkirk and District Royal Infirmary, Major's Loan, Falkirk FK1 5QE, UK

Summary: Two cases are described in which cardiac ischaemia presented with pain confined to the ear. In both cases the correct diagnosis was missed and treatment delayed. Angina and myocardial infarction may present with pain in the ear due to referral via the vagus nerve.

\section{Introduction}

Recent advances in the treatment of unstable angina and myocardial infarction have increased the need for early and accurate diagnosis. In the absence of chest pain, discomfort in the arms, back, neck or jaw, associated symptoms such as nausea, sweating and dyspnoea, suggest cardiac ischaemia. Two cases are presented in which referral of ischaemic pain was confined to the ear. In both cases the pain was attributed to local pathology, despite associated symptoms compatible with cardiac ischaemia.

\section{Case 1}

A 52 year old woman presented with a 4 hour history of acute onset severe aching pain in her right ear associated with nausea and sweating. The pain was localized within the ear and did not radiate. No discomfort was felt in the chest, arms, neck or face. There was no past history of similar pain or of ear disease. There was no history of ischaemic heart disease.

On admission she was pale and distressed. Her pulse was $100 /$ minute, in sinus rhythm, blood pressure was $140 / 90 \mathrm{mmHg}$, jugular venous pressure was raised $4 \mathrm{~cm}$, and heart sounds were normal. Her right ear appeared normal and was not tender. Auroscopic examination of both ears was unremarkable and hearing was normal.

The ear pain gradually settled with oral analgesia. The following morning an electrocardiogram

Correspondence: P.M. Rothwell, M.R.C.P., Western General Hospital, Crewe Road, Edinburgh EN4 2XU, UK.

Accepted: 26 October 1992
(ECG) was performed, as was routine on all admissions, and showed an evolving inferior myocardial infarct. Thrombolytic therapy was initiated 10 hours after admission. Cardiac enzymes were normal in blood taken on admission, but were raised in a sample taking 9 hours later; creatine kinase 1,136 IU/1 (normal 23-142 IU/1); aspartate aminotransferase $116 \mathrm{IU} / 1$ (12-31 IU/1); lactate dehydrogenase 394 IU/1 (195-415 IU/1). Despitefurther enquiry she denied any discomfort in he? chest, arms, neck or jaw. She made an uncomplicated recovery with no residual ear pain.

\section{Case 2}

A 65 year old man presented with a 10 day history of exertional pain in his right ear. He described an aching pain within the ear associated with mild dyspnoea. He denied any symptoms of recent upper respiratory tract infection, and had no past history of ear disease. He had experienced no pain or discomfort in his chest, arms, neck or jaw. On consulting his general practitioner he was prescribed gentamicin ear drops. On the day of admission he developed right ear pain at rest. The pain lasted one hour and was associated with a feeling of chest tightness.

On admission his pulse was $78 /$ minute sinus rhythm, blood pressure was $160 / 100 \mathrm{mmHg}$, heart sounds were normal and chest was clear. Examination of both ears was unremarkable.

An admission electrocardiograph showed ST depression in leads II, III, AVF, V5 and V6. He was treated with aspirin $150 \mathrm{mg}$ oral and intravenous infusions of nitrate and heparin. An ECG performed 2 hours later showed complete resolution of the ischaemic changes. Cardiac enzymes were nor- 
mal. He made an uncomplicated recovery with no recurrence of pain in his ear or chest.

\section{Discussion}

Cardiac pain is commonly referred to the arms, neck or lower jaw, but may also be felt in the face, back, occiput, or throat. ${ }^{1}$ Although such referred pain is usually accompanied by chest discomfort, ${ }^{1}$ it may occasionally occur in isolation. Referral of cardiac pain to the ear is rare, and there are no reports to our knowledge of pain confined to the ear.

Cardiac pain is predominantly transmitted via the first five thoracic sympathetic roots. ${ }^{2}$ This accounts for the referral of pain to the chest wall and arms, but not the face or jaw. Cardiac sympathectomy has been used to treat angina, ${ }^{3}$ but pain felt in the face and jaw is not helped. However, such pain may be abolished by section of the vagus. ${ }^{4}$ Alcohol block of the branches of the trigeminal nerve may also abolish angina pain felt above the neck. ${ }^{5}$ Connections between the vagus and the trigeminal nerve nuclei ${ }^{6}$ may explain the referral of pain to the face and jaw. The vagus also provides a route for referral of pain to the ear. In

\section{References}

1. Sampson, J.J. \& Cheitlin, M.D. Pathophysiology and differential diagnosis of cardiac pain. Prog Cardiovasc Dis 1971, 13: 507-531.

2. Malliani, A. \& Lombardi, F. Consideration of the fundamental mechanisms eliciting cardiac pain. Am Heart J 1982, 103: 575-578.

3. Leriche, R. \& Fontaine, R. Surgical treatment of angina pectoris. Am Heart J 1927, 3: 649-671.

4. Eppinger, H. \& Hofer, G. Zur pathogenese und therapie der angina pectoris. Therap Gegenw 1923, 64: 166-170.

5. Lindgren, I. \& Olivercrona, H. Surgical treatment of angina pectoris. J Neurosurg 1947, 4: 19-39. addition to carrying visceral afferent fibres from the chest and abdomen, the vagus also supplies sensation to the external auditory meatus, via the nerve of Arnold. Referral of pain via this pathway explains why bronchogenic carcinoma, ${ }^{7}$ oesophagitis $^{8}$ and aortic dissection, ${ }^{9}$ as well as angina and myocardial infarction, may cause pain in the ear. This link between the thoracic viscera and the ear also works in reverse, as seen when irritation of the external auditory meatus during auroscopy causes coughing, bradycardia and syncope.

In both these cases the correct diagnosis was missed because of the atypical site of the pain. In the first patient the acute onset of nausea and sweating should have suggested the possibility of 'silent' myocardial infarction, but the presence of ear pain suggested local pathology. It is important to recognize that pain in the ear may indicate cardiac pathology.

\section{Acknowledgements}

I would like to thank Dr S.K. Ghosh, Consultant Physician, Middlesbrough General Hospital, and Dr P. McSorley, Consultant Physician, Falkirk and District Royal Infirmary, for allowing me to report their patients.

6. Guyton, A.C. Structure and Function of the Nervous System, 2nd ed. W.B. Saunders, Philadelphia, 1976.

7. Bindoff, L.A. \& Heseltine, D. Unilateral facial pain in patients with lung cancer: a referred pain via the vagus. Lancet 1988 , i: 812-815.

8. Blau, J.N. Ear pain referred by the vagus. $B r M e d J 1989$, 299: $1569-1570$.

9. Hoffbrand, B.I. \& Hollman, A. Pain in the ear - a presenting symptom of aortic dissection. Br Med J 1985, 290: 1112. 